\title{
Farnesoid X Receptor (FXR) Interacts with Camp Response Element Binding Protein (CREB) to Modulate Glucagon-Like Peptide-1 (7-36) Amide (GLP-1) Secretion by Intestinal $L$ Cell
}

Pengzhou Li Liyong Zhu Xiangwu Yang Weizheng Li Xulong Sun Bo Yi Shaihong Zhu 


\section{Retraction Statement}

Li P, Zhu L, Yang X, Li W, Sun X, Yi B, Zhu S, entitled "Farnesoid X Receptor (FXR) Interacts with cAMP Response Element Binding Protein (CREB) to Modulate Glucagon-Like Peptide-1 (7-36) Amide (GLP-1) Secretion by Intestinal L Cell" [Cell Physiol Biochem 2018;47:1442-1452, DOI: 10.1159/000490836].

Herewith we withdraw our manuscript

All experiments in our manuscript were performed by our own team independently and all the data obtained were original. All experimental records and raw data have been kept for review.

However, the source of one of the cell lines we used in the study, the GLUTag cell line, is unknown. The source is unauthorized and we are unable to clarify the true source of the cell line. Therefore, we are trying to contact the author who first described the cell line to obtain an authorization.

We are willing to withdraw our manuscript because of unknown source of the cell line. We sincerely apologize for our mistake. 